\title{
Puilaurens (Puylaurens)
}

Château de Puilaurens (Puylaurens)

Lucien Bayrou

\section{(2) OpenEdition}

Édition électronique

URL : http://journals.openedition.org/adlfi/11884

ISSN : 2114-0502

Éditeur

Ministère de la culture

Référence électronique

Lucien Bayrou, «Puilaurens (Puylaurens) », ADLFI. Archéologie de la France - Informations [En ligne] Languedoc-Roussillon, mis en ligne le 01 mars 2004, consulté le 20 avril 2019. URL : http:// journals.openedition.org/adlfi/11884

Ce document a été généré automatiquement le 20 avril 2019.

(C) Ministère de la Culture et de la Communication, CNRS 


\section{Puilaurens (Puylaurens)}

Château de Puilaurens (Puylaurens)

\section{Lucien Bayrou}

\section{Identifiant de l'opération archéologique :}

Date de l'opération : 1994 - 1998 (SD)

Inventeur(s) : Bayrou Lucien

1 Vers le milieu du XIII ${ }^{e}$ s., Puilaurens (Puylaurens) appartient au roi de France qui en ordonne l'évacuation et la fortification en 1255. Forteresse royale, Puilaurens (Puylaurens) est réparé dans les années 1393-1394. Au cours des guerres francoespagnoles en 1473 et en 1495, il résiste à deux sièges.

2 L'accès s'effectue côté ouest au moyen d'une rampe d'accès en chicane. Le château se compose de deux enceintes accolées (Fig. n¹: Plan du château de Puilaurens), dont le tracé irrégulier épouse strictement le contour du rocher. La première enceinte est fermée par des courtines conservant encore des vestiges du crénelage. Une tour semi-circulaire, ouverte à la gorge, s'élève à l'angle sud-est. Une autre tour de plan semblable flanque le milieu du front sud. Dominant cette première enceinte, la seconde enceinte s'organise autour d'une tour quadrangulaire. Une tour lisse, ouverte à la gorge, occupe l'angle nord ; une autre à bossages, couverte par une voûte d'ogives, domine les chicanes. Citerne, latrines et mâchicoulis complètent cette deuxième enceinte.

\section{Les sondages de 1994}

3 Les sondages ont eu pour objet l'évaluation du site, en relation avec les travaux de consolidation et de restauration, effectués par la conservation régionale des Monuments historiques et dirigés par Monsieur l'architecte en chef des Monuments historiques.

4 Après dégagement d'une partie des chicanes d'accès, aux endroits les plus engraissés par les remblais, nous avons mis au jour les aménagements du sentier, matérialisés soit par le 
rocher taillé, soit par quelques rares vestiges de calade réalisée en pierres posées de chant arrondies par l'usage. Le réduit d'entrée, ménagé entre les deux portes de la première enceinte, a été dégagé.

5 Dans la première enceinte, des sondages ont été effectués à l'emplacement de la prise de terre du paratonnerre. L'observation du comblement traduit un endroit utilisé en décharge, car cette zone se situe à l'aplomb d'une fenêtre de la seconde enceinte.

6 Un autre sondage a été réalisé de part et d'autre de l'emplacement d'un mur de refend volontairement démoli à l'époque moderne. Le sol extérieur de ce bâtiment, ainsi délimité, semble avoir été partiellement aménagé en passage.

7 À l'extérieur de la première enceinte, à l'aplomb de la poterne nord, les vestiges d'une citerne extérieure ont été mis en évidence.

Différents éléments de mobilier métallique d'époque médiévale ou moderne - carreau d'arbalète, boulets, etc. -, des tessons de céramique locale à cuisson réductrice ou oxydante glaçurée, ainsi que quelques fragments de céramique d'importation espagnole des $\mathrm{XV}^{\mathrm{e}} \mathrm{s}$. et $\mathrm{XVI}^{\mathrm{e}} \mathrm{s}$. ont été découverts.

\section{Les sondages de 1995}

Dans la première enceinte, les résultats du sondage réalisé à l'aplomb du mur fermant la gorge de la tour sud, montrent le rocher, soigneusement taillé en emmarchement permettant l'accès à l'intérieur.

10 Le bâtiment, situé à l'ouest de l'escalier menant au chemin de ronde de la courtine, est construit en même temps que celle-ci, comme l'indique son arrachement. Une porte ménagée à l'angle nord-ouest, entre le bâtiment proprement dit et la falaise supportant la seconde enceinte, donne accès à un espace libre circonscrit entre rocher et courtines, utilisé comme décharge.

11 À une époque tardive, le bâtiment probablement en ruine est démoli, ses matériaux sont alors récupérés et utilisés dans la construction (bâtiment sud) ou le surhaussement (bâtiment nord) des deux corps d'habitation bordant la cour. L'ensemble du mobilier archéologique mis au jour dans la zone date d'une période variant du XV $\mathrm{X}^{\mathrm{e}} \mathrm{s}$ aux XVII $\mathrm{e}$. et XVIII ${ }^{\mathrm{e}}$ s., époque vraisemblable de la démolition de cette structure.

Dans la seconde enceinte, l'étude du comblement montre la complexité de la stratigraphie et la difficulté d'interprétation de l'espace situé entre la citerne et la tour nord-ouest. À un sol d'époque médiévale, que l'on peut situer au niveau de la gargouille, succède un hérisson de pierre tout-venant mêlée de tessons de tuiles, sur lequel est construit le mur de refend tardif (probablement du XVII ${ }^{e}$ s.), s'élevant au nord. Ce dernier condamne la volée d'escalier plaquée au revers de la courtine nord-ouest. Contre le mur de refend, une sorte de banquette semble avoir été aménagée, soit en vue d'une utilisation, soit plus probablement pour le conforter. Au sud, vers l'angle sud-ouest de la citerne, appuyés contre le rocher taillé, les vestiges d'un massif d'escalier matérialisent le nouvel accès.

On peut poser l'hypothèse, confirmée par l'étude historique d'une occupation et de la mise en défense du château de Puilaurens (Puylaurens) aux XVI ${ }^{\mathrm{e}}$ s. et XVII ${ }^{\mathrm{e}}$ s., impliquant une utilisation plus longue que le site voisin de Peyrepertuse par exemple. 


\section{Les sondages de 1996}

14 L'exploration archéologique de campagne de 1996 a eu pour objet essentiel la fin de l'étude du comblement de la zone située dans la seconde enceinte et circonscrite par la tour ouest, la courtine sud, la citerne et le mur récent nord.

15 La poursuite du décapage a permis de dégager le niveau du sol d'utilisation, situé à un niveau inférieur à $1,10 \mathrm{~m}$ du seuil de la porte de la tour. Différents ensembles ont été repérés:

- un évier, composé d'une pierre de taille, ménagé sur le retrait de l'empattement de la fondation de la courtine sud se prolongeant vers l'extérieur par une gargouille ;

- une structure matérialisée par une pierre taillée en disque (d'un diamètre de $0,57 \mathrm{~m}$ ), encastrée dans le rocher et sertie par un bourrelet de mortier de chaux. L'aspect rubéfié du mortier et du disque fait penser à l'emplacement d'un foyer.

16 Un effondrement du substrat a permis l'observation des solutions techniques adoptées par les constructeurs face aux difficultés présentées par le caractère faillé du rocher calcaire servant d'assiette à la forteresse. Une faille étroite et profonde a ainsi été obturée par des quartiers de rocher, sur lesquels reposent des déchets de taille et des gravats provenant du chantier de construction lui-même, de façon à obtenir un sol d'utilisation horizontal.

\section{ANNEXES}


Fig. $n^{\circ} 1$ : Plan du château de Puilaurens

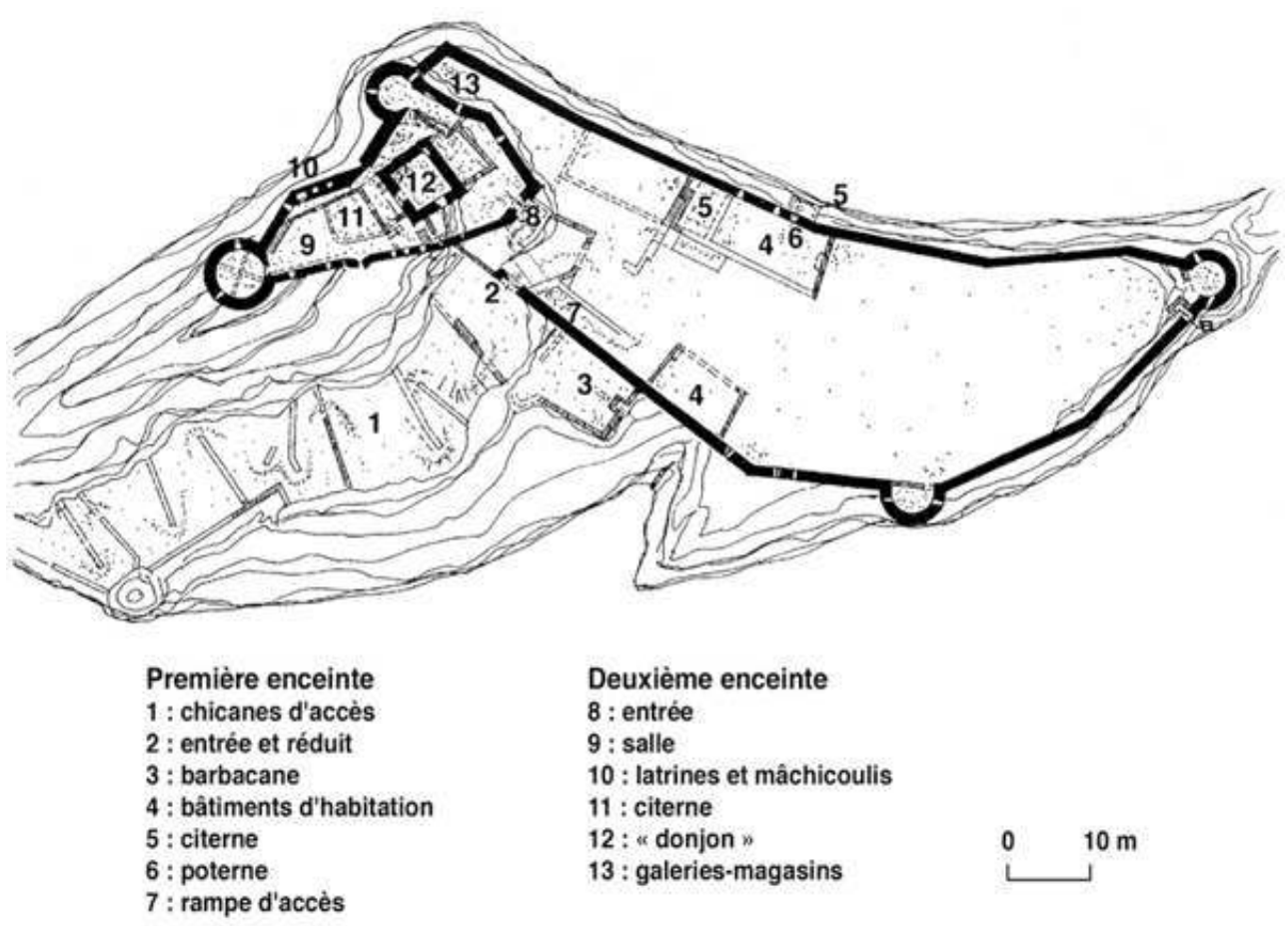

Auteur(s) : Bayrou, Lucien. Crédits : ADLFI - Bayrou, Lucien (2003)

INDEX

Index chronologique : ép médiévale

Index géographique : Languedoc-Roussillon, Aude, Puilaurens operation sondage (SD) 